\title{
PeneloPET v3.0, an improved multiplatform PET Simulator
}

\author{
A.Lopez-Montes, J.L. Herraiz, P. Galve, S. España, E.Vicente, J. Cal-Gonzalez, J.M. Udias
}

\begin{abstract}
PeneloPET is a Monte Carlo simulation tool for positron emission tomography based on PENELOPE. It was developed by the Nuclear Physics Group at University Complutense of Madrid and its initial version was released in 2009. In this work, we present PeneloPET v3.0, which is now available precompiled for Microsoft Windows, MacOS and Linux OS. This new release includes improved simulations of the positron range in different materials and an accurate description of the decay cascades for many radioactive nuclei including the most common non-pure positron emitters used in PET. This enables the simulation of PET acquisitions with positron-gamma emitters. This release also includes many different fully-working examples, of both clinical and preclinical scanners, as well as several numerical phantoms. Due to the simplicity of the input the output files, and the installation process, PeneloPET v3.0 can be perfectly used not only for research, but also as an educational tool in class.
\end{abstract}

Key words - Monte Carlo simulations, Positron Emission Tomography, PENELOPE.

\section{INTRODUCTION}

PeneloPET[1] is a Monte Carlo simulation tool[1,2] for positron emission tomography (PET) based on PENELOPE[3]. It was first released in 2009 by the Nuclear Physics Group at Complutense University of Madrid. Since the first release, some features have been improved and added, making PeneloPET v3.0 more user-friendly, faster and with improved physical considerations which make the simulations more realistic and useful. These new features comprise of improved simulations for positron range for different materials and isotopes[4], a detailed simulation for self coincidence detection[5] including the case of the inner activity of the crystals of the scanner as well as the possibility of simulating non-pure beta emitters and multiple gamma emissions[6], incorporating the possibility of including decay cascades for the nuclei and providing more realistic simulations.

This release includes a library with many examples for geometries similar to the main current PET scanners, both for preclinical and clinical PET. Moreover, this version has been compiled and released also for multiplatform, which make PeneloPET v3.0 more accessible to the user. In this work, we

This is a contribution to the Moncloa Campus of International Excellence. Part of the calculations of this work were performed in the "Clúster de Cálculo para Técnicas Físicas" funded in part by UCM and in part by UE Regional Funds. We acknowledge support from the Spanish Government (FPA2015-65035-P, RTC-2015-3772-2), from Comunidad de Madrid (S2013/MIT-3024 TOPUS-CM, B2017/BMD-3888 PRONTO-CM) and European Regional Funds. This work is also supported by EU's H2020 under MediNet, a Networking Activity of ENSAR-2 (grant agreement 654002). This work is also supported by NIH R01 CA215700-2 grant. present the main improvements and additions since the first release of PeneloPET and which are included in PeneloPET v3.0 release.

\section{II.NEW FEATURES}

\section{A. Multiplatform version and simplified input/output}

PeneloPET v3.0 release has been compiled and distributed for Microsoft Windows, Linux OS and Mac OS platforms, which make the use of PeneloPET v3.0 much easier. Some inputs and outputs have been reduced and simplified as well in order to make PeneloPET more user-friendly and for a proper compilation for multiplatform.

PeneloPET v3.0 also includes a tool to generate sinograms from the coincidence output file with the specified usual parameters (span, maximum ring-difference, segments...)

\section{B. Improved simulation of positron range}

In PeneloPET v3.0, new parametrized models of the positron range distribution for different materials and isotopes are included. The range profiles of the main $\beta^{+}$emitters used in PET as ${ }^{18} \mathrm{~F},{ }^{11} \mathrm{C},{ }^{13} \mathrm{~N}$ or ${ }^{15} \mathrm{O}$ and some other $\beta^{+}$emitters as ${ }^{82} \mathrm{Rb},{ }^{124} \mathrm{I}$ or ${ }^{68} \mathrm{Ga}$ are provided for some of the most important materials in a usual PET study as water or cortical bone. More range profiles for different materials and isotopes can be easily generated using PeneloPET v3.0.

\section{C.Improved description of decay cascades for non-pure $\beta^{+}$} emitters.

PeneloPET v3.0 incorporates the possibility of including decay cascades of the nucleus. This feature allows the definition and the realistic simulation for complex isotopes with different decaying modes and for nonpure $\beta^{+}$emitters. This is very useful to simulate triple coincidences (two photons from the positron annihilation and another gamma emission from the nucleus). The different branching ratios and the particles emitted in each decaying process including the energy of these particles can be easily defined in the input files.

\section{D.Intrinsic activity of ${ }^{176} \mathrm{Lu}$}

Most current PET scanners use crystals with Lutetium (LSO or LYSO) because of their good physical properties. However, the intrinsic activity of natural ${ }^{176} \mathrm{Lu}$ yields several prompt gamma rays in cascade, with energies of 88, 202 and $307 \mathrm{keV}$. This generates a background of spurious coincidences[5]. PeneloPET 3.0 can simulate properly the internal activity of the crystals used in the scanner and the background of coincidences that they generate. 


\section{VALIDATION OF THE NEW FEATURES FOR DIFFERENT SCANNER GEOMETRIES}

PeneloPET v3.0 has been tested using many different cases, and it includes a large library of scanner configurations emulating the most commonly used ones in preclinical and clinical imaging. For example, in [2], it was evaluated against the experimental values of sensitivity and NEC rates of several Biograph PET/CT scanners. In Fig. 1, we present a representation using gview3d[3] for some scanner geometries included in the examples of the new release of PeneloPET v3.0. Some inputs and outputs of the simulations corresponding to the geometries presented in Fig. 1 are shown in Figs. 2-5.
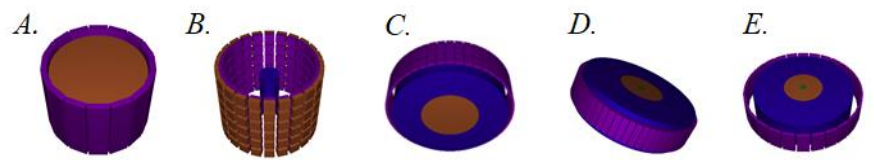

Fig. 1. Representation using gview3d[3] of simulated scanners with different geometries based on commercial PET scanners. A. INVEON preclinical scanner (Siemens). B. SUPERARGUS PET/CT preclinical scanner 6-rings version (SEDECAL). C. Biograph TPTV PET/CT clinical scanner (Siemens). D. Discovery PET/CT clinical scanner (GE). E. Ingenuity PET/CT clinical scanner (Phillips). Different environments (objects) for the simulations are also shown. Each color in the figure represents a different material in the simulation.

\section{A. INVEON preclinical scanner \\ Transverse Coronal Transverse Coronal
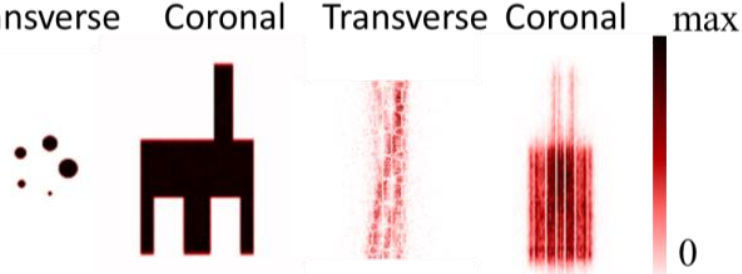

Fig. 2. (Left) Image of decays provided by PeneloPET during the simulation This image corresponds to a total of above $6 \cdot 10^{8}$ decay processes. This simulation has been performed from a distribution of sources representing an IQ NEMA phantom for a mouse size. (Right) Sinogram of true detections generated by the sinogram functionality distributed with PeneloPET 3.0. This sinogram corresponds to a total of $6.77 \cdot 10^{6}$ detected counts with 175 radial bins and 128 angular bins. SSRB has been applied to obtain a rebinned sinogram.

\section{B. SUPERARGUS preclinical scanner}

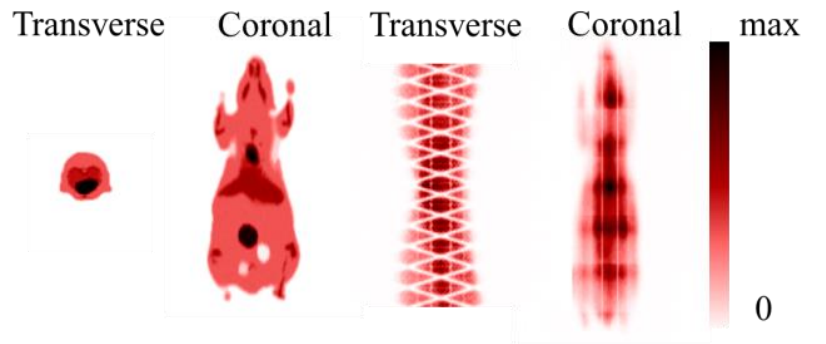

Fig. 3. (Left) Image of decays provided by PeneloPET during the simulation. This image corresponds to a total of above $5 \cdot 10^{8}$ decay processes. This simulation has been performed from a numerical phantom representing the uptake of FDG in a mouse. (Right) Sinogram of true detections generated by the sinogram functionality distributed with PeneloPET 3.0 with a total of $1.36 \cdot 10^{7}$ detected counts with 175 radial and 128 angular bins. SSRB has been applied to obtain a rebinned sinogram.

\section{Biograph clinical scanner}

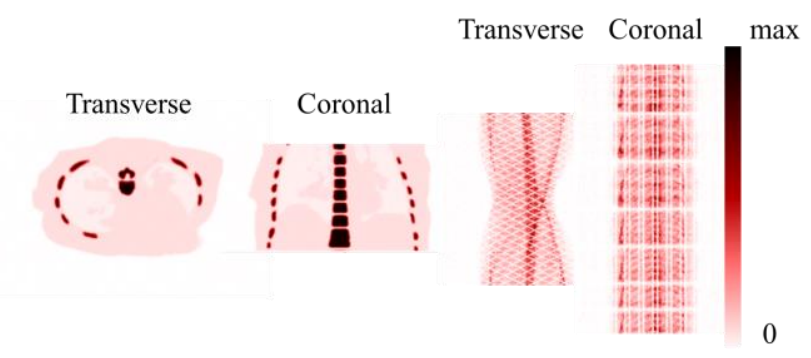

Fig. 4. (Left) Voxelized source obtained from a numerical phantom used for the simulation of the uptake of $\mathrm{NaF}$ in a human torso. (Right). Sinogram of true detections generated by the sinogram functionality distributed with PeneloPET 3.0. This sinogram corresponds to a total of $4 \cdot 10^{6}$ detected true counts with 336 radial bins and 336 angular bins. 3D sinogram with no rebinning is shown and the different segments can be appreciated.

\section{Other clinical scanner}

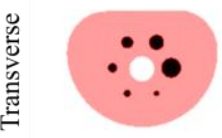

Discovery (GE) Ingenuity (Phillips)
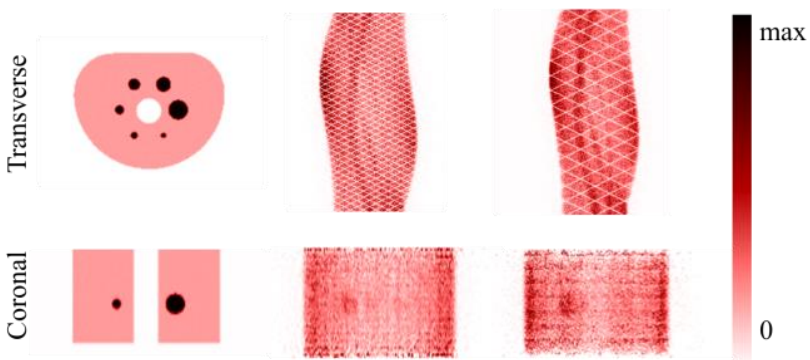

Fig. 5. (Left) Image of above $6 \cdot 10^{8}$ decay processes provided by PeneloPET during the simulation of the activity distribution of ${ }^{18} \mathrm{~F}$ in an IQ NEMA phantom for clinical scanners. (Middle) Sinogram for Discovery PET/CT scanner (GE) geometry of true detections generated by the sinogram functionality distributed with PeneloPET 3.0 with a total of $2.49 \cdot 10^{6}$ detected counts with 300 radial and 320 angular bins. (Right) Sinogram for Ingenuity PET/CT scanner (Phillips) of true detections. 1.59.10 detected counts in the sinogram with 480 radial and 336 angular bins. SSRB has been applied to obtain a rebinned sinograms.

\section{CONCLUSIONS}

In this work, the new features and improvements of the new release of the Monte Carlo PET simulator PeneloPET are presented. It provides a large library of examples, improved physical considerations and the possibility of using PeneloPET in multiple OS.

\section{REFERENCES}

[1] S.España et al. "PeneloPET, a Monte Carlo PET simulation tool based on PENELOPE: features and validation". Physics in Medicine \& Biology, 54(6), 1723. 2009

[2] S.Jan et al. "GATE V6: a major enhancement of the GATE simulation platform enabling modelling of CT and radiotherapy." Physics in Medicine \& Biology 56.4, 881. 2011

[3] F.Salvat, J.M.Fernández-Varea, and J. Sempau. "PENELOPE-2008: A code system for Monte Carlo simulation of electron and photon transport." Workshop Proceedings (Vol. 4, No. 6222, p. 7). July 2006.

[4] J.Cal-González et al. "Positron range estimations with PeneloPET." Physics in Medicine \& Biology 58.15, 5127. 2013

[5] M.Conti, L.Eriksson, H.Rothfuss, T.Sjoeholm, D. Townsend, G.Rosenqvist, T.Carlier. "Characterization of 176Lu background in LSO-based PET scanners". Physics in Medicine \& Biology, 62(9), 3700. 2017

[6] J.Cal-González et al. "Simulation of triple coincidences in PET." Physics in Medicine \& Biology 60.1, 117. 2014

[7] K.M.Abushab et al. "PeneloPET simulations of the Biograph ToF clinical PET scanner." 2011 IEEE Nuclear Science Symposium Conference Record. IEEE, 2011 